\title{
Meta Concrete: Exploring Novel Functionality of Concrete Using Nanotechnology
}

\author{
Yining Feng, ${ }^{1}$ Yenfang Su, ${ }^{1} \mathrm{Na} \mathrm{Lu}{ }^{1,2^{*}}$ and Surendra Shah ${ }^{3,4^{*}}$
}

Meta-concrete refers to the cementitious composites that possess novel functionality and superior performance through advanced manufacturing process, such as nanotechnology. Modern concrete bears both structural and functional features, the former of which has been intensively researched in the last decades, but multi-functional features of concrete structures related to heat, light, sound, vibration, etc. have not been systematically investigated. In this paper, the recent development of multi-functional concrete, also known as meta-concrete, is reviewed with focus on the improvement of traditional properties, such as mechanical and transport properties, and endorsement of the novel functionalities such as sensing, self-cleaning, energy harvesting by taking advantage of nanotechnology in cement and concrete. Besides the addition of nanoparticles into cement concrete to make it multifunctional, a new technique of developing silica-based hybrid nanocomposites for surface treatment of concrete to achieve novel functions is reviewed and its feasibility is discussed. This work could shed light on the future development of meta-concrete.

Keywords: Nano-engineered meta-concrete; Modulus of elasticity; Durability; Photocatalytic; Thermoelectric; Piezoelectric

Received 16 October 2019, Accepted 19 November2019

DOI: $10.30919 / \mathrm{es} 8 \mathrm{~d} 816$

\section{Introduction}

Concrete is the most widely used construction material due to its high stability, availability and low cost. Structural properties of concrete such as strength, ductility, elasticity, creep resistance, and durability, have been intensively-studied; while other functionalities, such as heat storage, acoustic shielding, weight reduction, have also been discussed but to a lesser degree to satisfy the various needs of modern society.

In recent years, the rapid revolution in nanotechnology opens up an exciting opportunity to equip concrete with novel functionality. It has been found that a small addition of nanoparticle can greatly enhance various properties of concrete ${ }^{1}$. However, most of the current work focuses on adding nanoparticles to improve the mechanical properties and the durability. The potential of design and manufacturing of concrete materials that are adaptive to environmental circumstances such as heat, light, sound, vibration, have not been well explored yet, which, however, is achievable through nanotechnology exhibited by recent researches. To this end, the focus of this article is on examining the recent advancement of using innovative processing at nanoscale to

\footnotetext{
${ }^{1}$ Lyles School of Civil Engineering, Purdue University, WestLafayette, IN, 47907, USA

${ }^{2}$ School of Materials Engineering, Birck Nanotechnology Center, Purdue University, WestLafayette, IN, 47907, USA

${ }^{3}$ Presidential Distinguished Professor, Department of Materials Science \& Engineering, University of Texas at Arlington, Arlington, TX, USA

${ }^{4}$ Department of Civil and Environmental Engineering,Northwestern University, Evanston, IL, USA

*E-mail: luna@purdue.edu; s-shah@northwestern.edu
}

obtain adaptive properties of concrete at micro-scale that enables novel functionality such as energy harvesting, sensing, high durability and self-cleaning etc. Meanwhile, the use of nanotechnology to achieve desired mechanical properties that can rarely been accomplished by normal techniques has been discussed. All these developments demonstrate the possibility of tailoring the materials properties of concrete for the targeted applications. We hope this article could serve as a steppingstone for meta-concrete, a new type of concrete that possesses advanced processing, greatly enhanced properties, and novel performances.

\section{Meta-concrete of high modulus of elasticity by nanofiber}

Despite our industry has made considerable progress in enhancing the compressive strength of concrete, the rapid development of tall buildings often requires concrete with a high modulus of elasticity (MOE) to limit lateral deformations and ensure safety and occupant comfort. An increase of MOE is typically achieved by the increase of the compressive strength that always results in cost increases. Using nanotechnology, it has been demonstrated that it is feasible to obtain a high MOE concrete without necessarily increasing the compressive strength, which is summarized in this section.

2.1 Effect of small addition of CNT/CNF on concrete flexural strength Many researchers have studied reinforcing cement composites with carbon nanotube and carbon nanofibers. When well dispersed, ${ }^{2,3}$ a very small amount of CNT or CNF (less than $0.1 \%$ by weight of cement) can substantially improve the flexural strength, reduce autogenous shrinkage, enhance piezo resistivity, and delay potential corrosion of reinforcing bars. For instance, the result of notched beam test has 


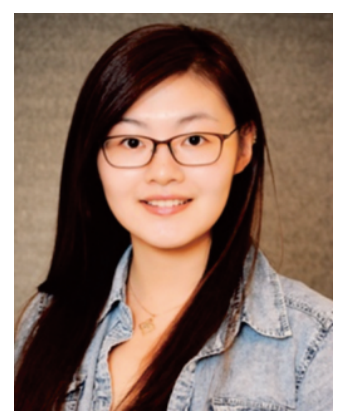

\section{Yining Feng}

Yining Feng is pursuing her Ph.D. degree of Civil Engineering at Purdue University. Her current research interests range from nanostructured oxides for high temperature thermoelectric applications to flexible thermoelectric devices for medium to low temperature applications. Yining has published one book chapter, 13 journal papers and three conference proceedings. Yining has attended the 2017 National Science Foundation (NSF) Innovation Corps (I-Corps) as an entrepreneur lead on high temperature thermoelectric technology development. This recognition led to an invitation to attend the Polsky Center for Women in STEM Leadership Workshop at the University of Chicago, sponsored by NSF.

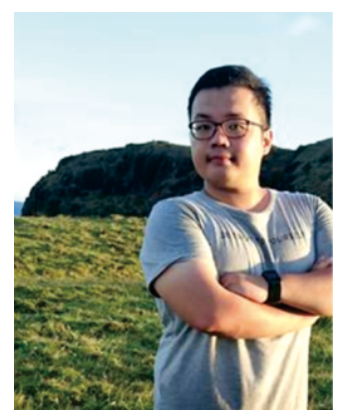

\section{Yenfang Su}

Yenfang Su is a Ph.D. student at the Lyles School of Civil Engineering. His research interests include developing advanced non-destructive testing (NDT) and sensing technology for civil infrastructure, innovative multifunctional sustainable cementitious composites, and emerging machine learning/data processing to predict the concrete properties. He was a structural engineer for few years, which led him equipped with strong mechanics background and practical engineering experiences. He is currently working on several exciting DOT (Department of Transportation) funded projects in development of in-situ sensing technology for concrete properties evaluation and designing the self-healing cementitious materials with ultra-high ductility.

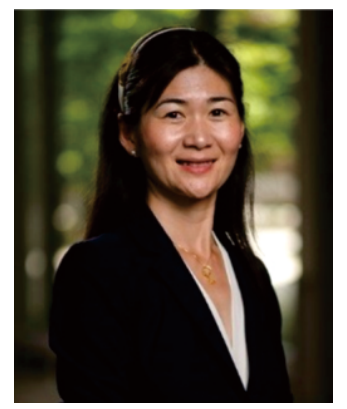

\section{Na (Luna) Lu}

$\mathrm{Na}$ (Luna) Lu is ACPA Rising Star Associate Professor at the Lyles School of Civil Engineering, and the School of Materials Science at Purdue University. Dr. Lu is the founding director of Center for Intelligent Infrastructure (CII) at Purdue University. Prof. Lu's research centers on the discovery of functional nanomaterials with tunable (mechanical, electrical, thermal, and optical) properties. She has worked across diverse research fields developing an interdisciplinary research program that integrates elements of material science, electrical engineering, and civil engineering. Such approach has led to many technological advances and innovations, which include high temperature thermoelectric materials for waste heat harvesting, multi-functional concrete for energy harvesting, piezoelectric materials for in-situ sensing of concrete properties, and advanced composites materials. Dr. Lu has won several scientific awards, including National Science Foundation (NSF) CAREER Award. She has also founded several spin-off companies with the technologies invented at her research lab.

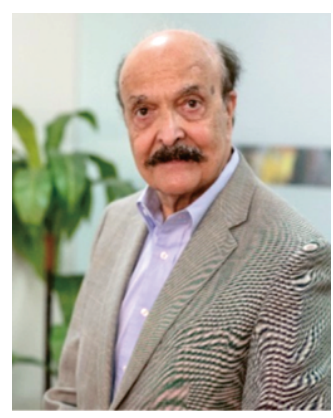

\section{Surendra P. Shah}

Professor Surendra P. Shah is a Presidential Distinguished Professor at University of Texas at Arlington and Walter P. Murphy Emeritus Professor of Civil Engineering at Northwestern University. He was the founding director of the pioneering National Science Foundation Science and Technology Center for Advanced CementBased Materials. His current research interests include: fracture, fiber-reinforced composites, nonde-structive evaluation, transport properties, processing, rheology, nano-technology, and use of solid waste materials. He has published more than 500 journal articles and edited more than 20 books, which has more than 60,000 citations. He is an honorary professor at several research university around world. Besides, Professor Shah is also a member of the US National Academy of Engineering, and foreign member of the Chinese Academy of Engineering, the Indian Academy of Engineering, Athens Academy and Russian Academy. 
indicated a substantial improvement in fracture properties of cement composites reinforced with CNT, as shown in Fig. 1.

\subsection{Effect of small addition of CNT/CNF on MOE}

With regard to the compressive strength, no noticeable influence is shown (Table 1) on cement mortar. However, it is interesting to note that the addition of CNT/CNF increases the modulus of cement mortar more than that of cement paste (Fig. 2, Table 1), and a desired MOE (about $50 \mathrm{GPa}$ ) of concrete can be achieved at a normal compressive strength level. This is unexpected since CNT (or CNF) can enhance the properties of cement matrix but they do not influence the properties of sand particles. This can be attributed to that CNT/CNF enhances the interface between sand (or coarse aggregates) and cement paste both chemically and physically. ${ }^{5}$ Conventional approach of reaching high MOE is often achieved by increasing compressive strength (about 150
$\mathrm{MPa}$ ), which makes concrete more brittle and requires high amount of cement. In contrast, a very high MOE is obtained with the addition of $\mathrm{CNT} / \mathrm{CNF}$ without the necessity of increasing compressive strength, as shown in Table 1 . The novelty of decoupling of strength and modulus has a great potential application for super tall buildings, sky scrapers.

Existing literature has indicated that addition of CNTs alters the nanostructure of calcium silicate hydrate (C-S-H). ${ }^{6,7}$ Researchers have also suggested that CNTs provide a massive surface area for the precipitation of cement hydrates, conceivably in a well-packed format that could contribute to the formation of denser C-S-H agglomerates with higher stiffness. ${ }^{8}$ This effect is shown in Fig. 3, where the results of nanoindentation tests on control paste specimens and specimens reinforced with $0.08 \%$ CNTs by weight of cement are presented as frequency plots of the calculated MOE values. The shift in the plots indicates that the addition of CNTs has increased the stiffness of the

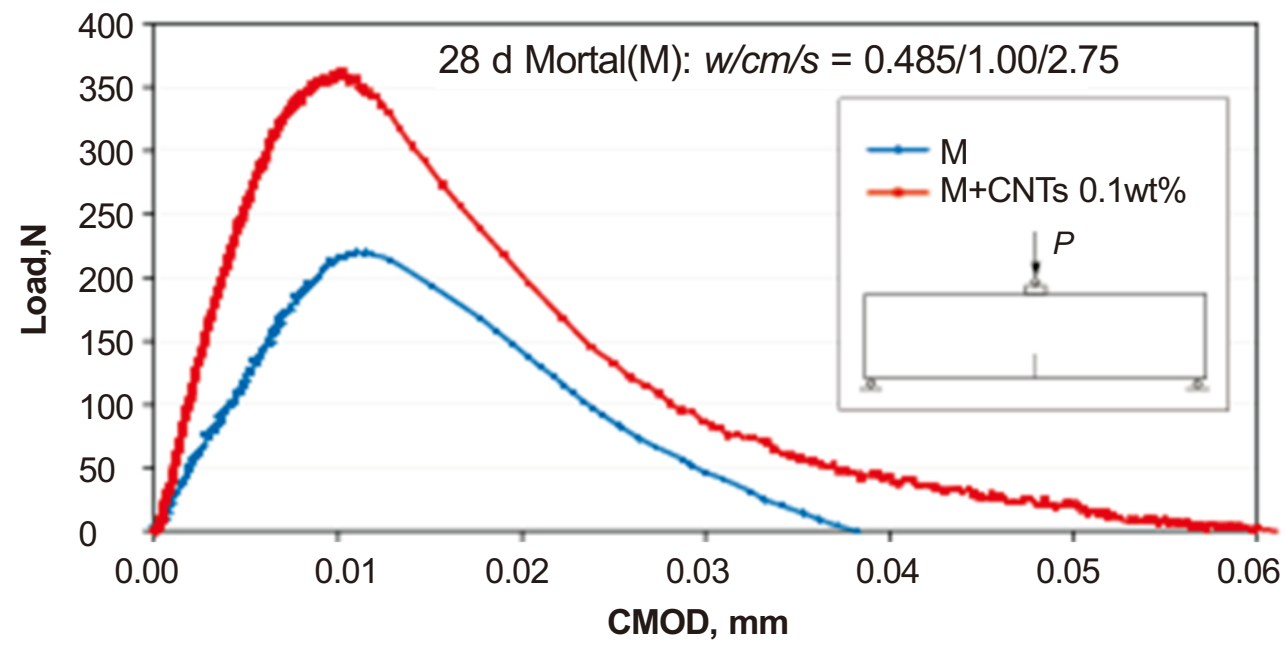

Fig. 1 Crack mouth opening displacement (CMOD) versus load for notched beam specimens fabricated from mortar (M) and mortar reinforced with $0.1 \%$ CNTs by weight of cement. ${ }^{4}$

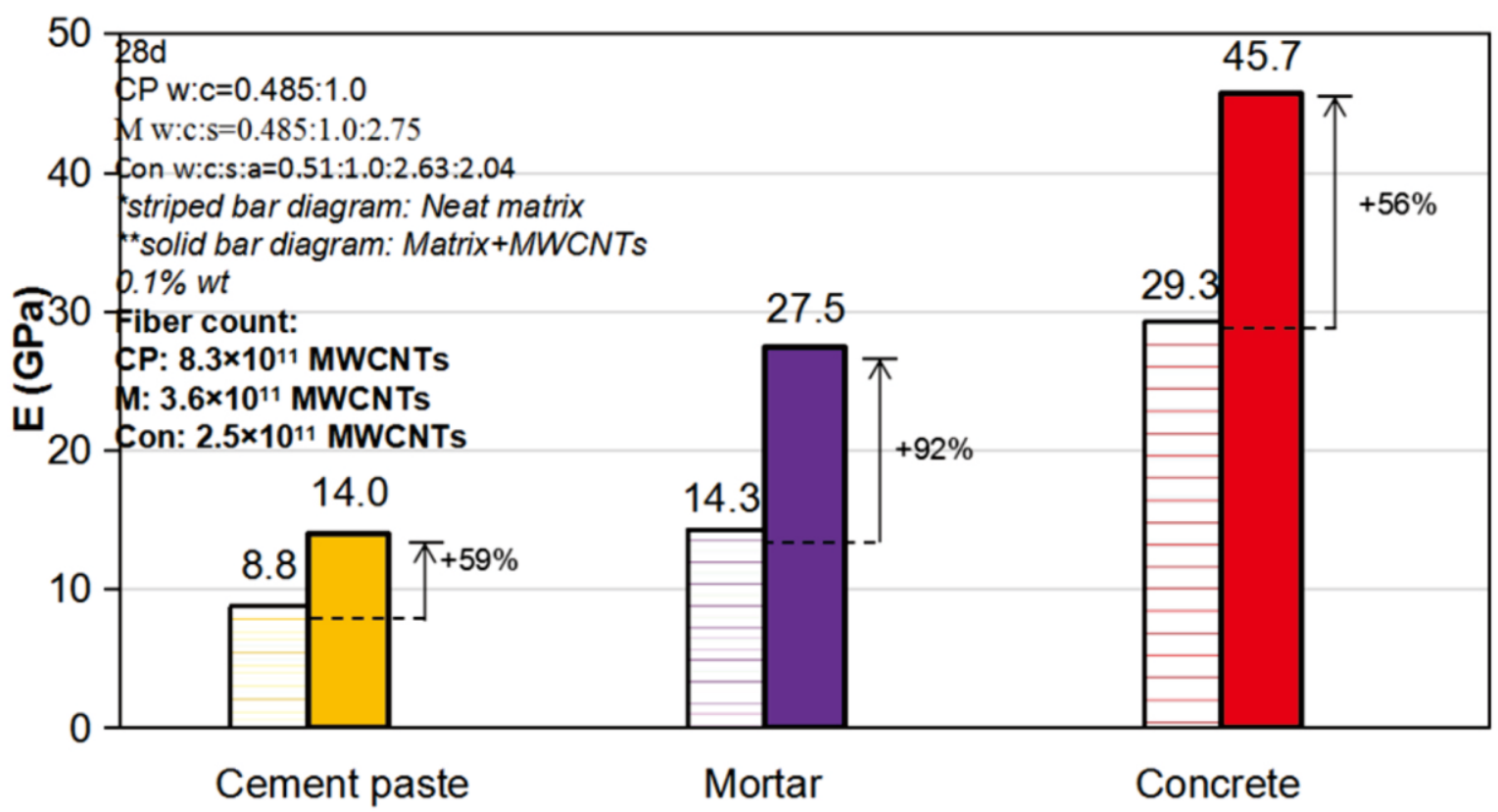

Fig. 2 28-day MOE for concrete and concrete reinforced with $0.1 \%$ CNTs by weight of cement. ${ }^{4}$ 
cement matrix.

Identifying the properties of the region where the cement paste meets the aggregate surface (interfacial transition zone, ITZ) is critical to the understanding of the strength and fracture behavior of $\mathrm{CNT} / \mathrm{CNF}$ reinforced cement concrete. To consider the overall interface effect, the thickness and the Young's modulus of ITZ are taken into account in an averaged manner. The PeakForce ${ }^{\mathrm{TM}} \mathrm{QNM}^{\mathrm{TM}}$
(Quantitative Nanomechanical Property Mapping) was used to study the Young's modulus of cement mortar, aggregate, and ITZ. As shown in Fig. 4, the QNM test results for the Young's modulus of cement mortar with and without CNF are $38.11 \mathrm{GPa}$ and $24.14 \mathrm{GPa}$, respectively, which are close to the macro-mechanical test results of $36.5 \mathrm{GPa}$ and $27.3 \mathrm{GPa}^{5}$.

Table 1 Mechanical properties of mortar/concrete with the addition of CNF.

\begin{tabular}{llll}
\hline & Fiber Count & $\begin{array}{l}\text { Compressive Strength } \\
(\mathrm{MPa})\end{array}$ & Young's Modulus (GPa) \\
\hline Plain Mortar & 0 & 40.6 & 27.3 \\
Mortar+CNF & $4.1 \times 10^{10}$ & 43.1 & 36.5 \\
Plain Concrete & 0 & 46.7 & 35.7 \\
Plain Concrete+CNF & $2.7 \times 10^{10}$ & 50.5 & 46.2 \\
\hline
\end{tabular}

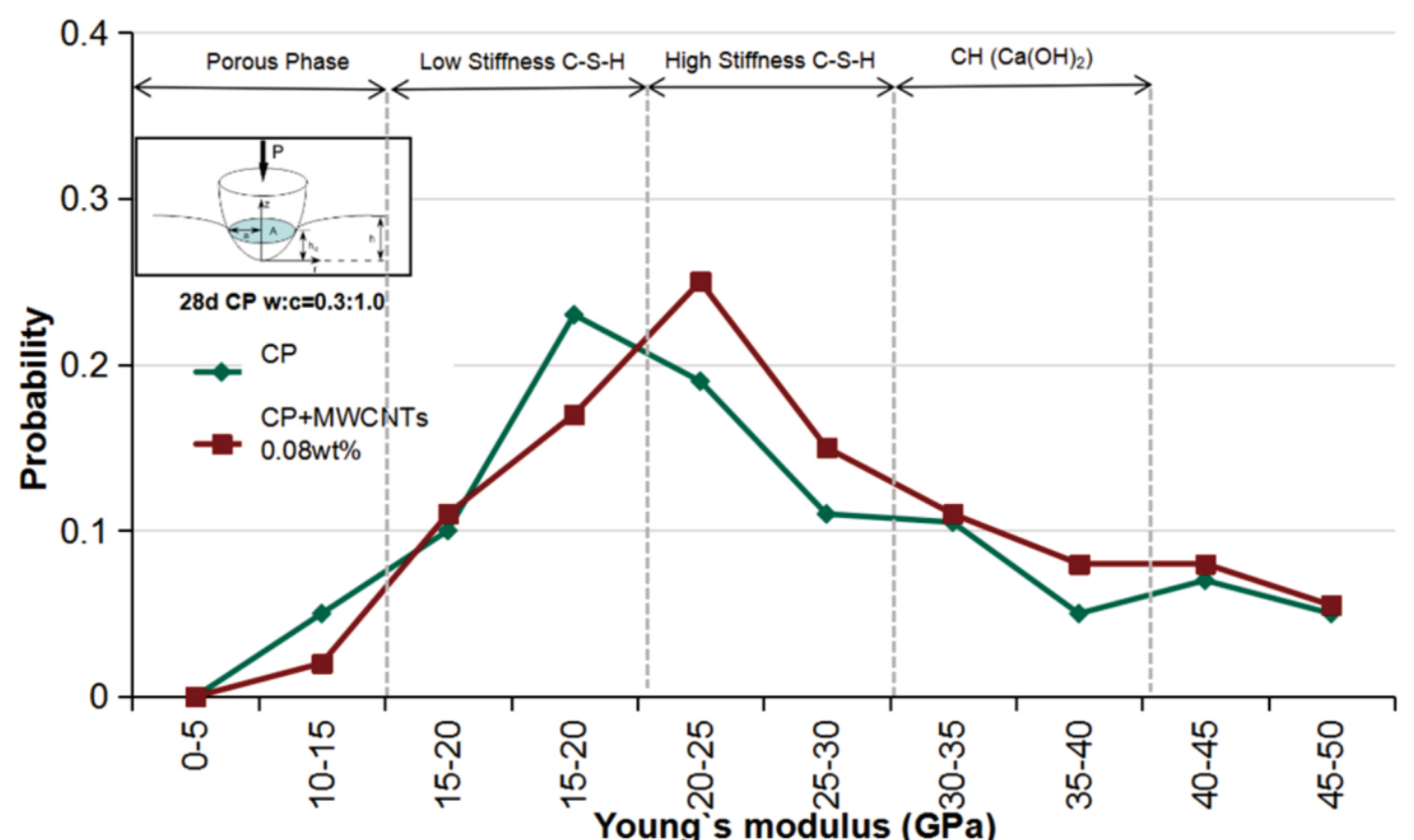

Fig. 3 Probability plots of the MOE for 28-day cement paste and cement paste reinforced with $0.08 \%$ CNTs by weight of cement.
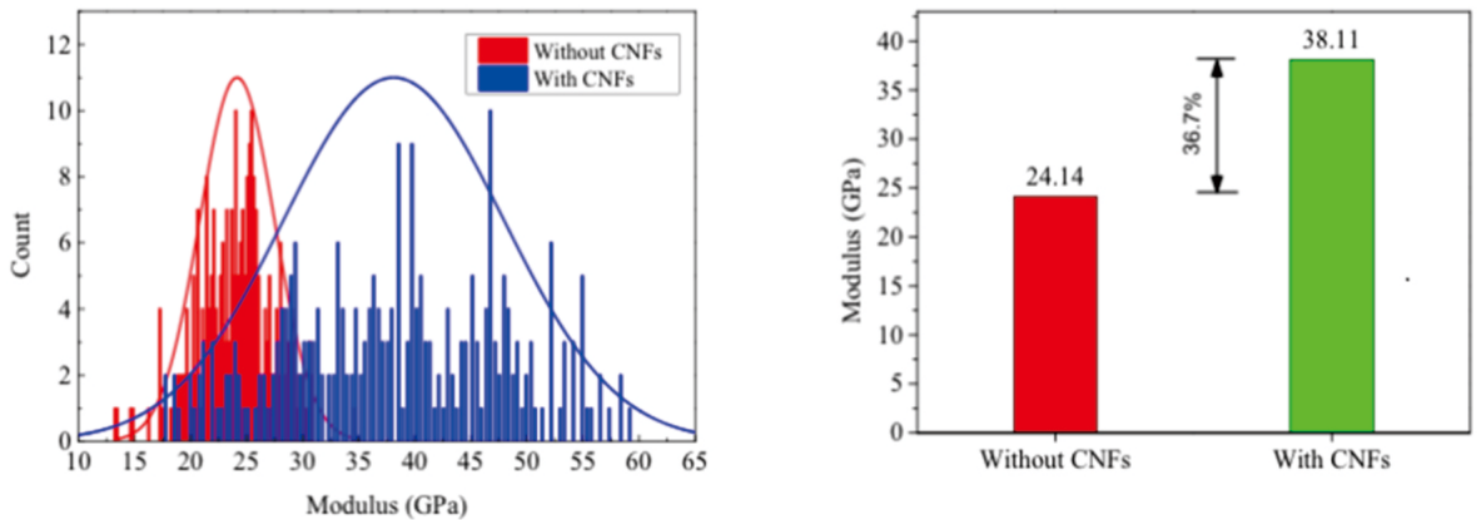

Fig. 4 QNM test results for Young's modulus of cement mortar with and without CNFs. ${ }^{5}$ 


\section{Meta-concrete of high durability achieved by surface coating}

Generally, the quality of the concrete surface greatly influences the corrosion resistivity of the structure and determines the service life. Moreover, the apparent features of concrete structures that impact the quality of living are predominately exerted by the surface, such as the decoration property, exchange of liquid/gas phases with the environment, acoustic absorption, etc. These functional features of the surface concrete are as important as the structural feature as they are directly related to people's daily life. Along this line, it is important to develop surface treatment techniques that can enhance the quality and/or enable novel functionalities of concrete structure.

\subsection{Surface coating with nanosilica for improved water resistivity}

The quality of concrete in service will always be affected by the environment, resulting a gradient porous microstructure from the surface, which in turn, accelerates the migration and deterioration processes of the surface. Surface treatment of concrete with different agents has always been used. Within them, organic agents, such as silane, siloxane and resin; inorganic agents, such as water glass, have been widely used in research and practice., ${ }^{9,10}$ Use of nanoparticle for surface treatment often encounters the issue of the penetration of nanoparticle into the concrete. Cardenas studied the electrokinetic-drive method for a high penetration of nanoalumina into cement concrete, which may not be easily used for other nanoparticles due to the requirement of the positive surface charge for a desired movement direction of the particle under an electrical field. Recently, silica-based nanomaterials have been used directly for surface treatment of hardened concrete, which shows the possibility of improving the quality of the surface by the design of the material and the treatment technique. ${ }^{12,13}$

When nanosilica is directly applied onto concrete surface by brushing technique, it turns out to be effective only in samples with large pore size. Hou et al. compared the water absorption reduction rate of different agents on hardened cement mortar. ${ }^{14}$ (Fig. 5) It can be seen that nanosilica does not show potential reduction of water absorption rate compared to the control sample even when the $\mathrm{w} / \mathrm{c}$ is relatively high. This implies that the penetration and filling of pores with nanosilica is no obvious even when its size is $20 \mathrm{~nm}$. However, it is found that the precursor of nanosilica, TEOS (tetraethoxysilane), greatly decreases the water absorption rate through its in-situ hydrolysis and reaction with the calcium hydroxide from cement hydration.

The findings indicate the potential of using nanosilica in reducing the transport properties of cement concrete. Furthermore, studies showed that the optimization of the microstructural and compositional properties of the surface concrete lead to the enhancement of the durability, such as calcium leaching resistivity, ${ }^{14}$ sulfate attack resistivity, ${ }^{14}$ chloride ion resistivity, ${ }^{15}$ carbonization resistivity. ${ }^{16}$

\subsection{Design of core-shell nanocomposites for hydrophobic/ superhydrophobic concrete surface}

Normally, there are two ways to improve the water resistivity of concrete, i.e., the densification of the porous microstructure and making the surface hydrophobic. It can be shown from section 3.1 that inorganic nanoparticles are capable of blocking the pores of concrete at the surface, showing the possibility for concrete coating. However, when comparing the water resistance with that of organic treatment agents, such as silane or siloxane, it still needs to be improved.

By taking the advantages of both the pore filling effect of nanoparticles and the lowing of the surface energy imposed by the organic treatment agents, a greatly reduced water absorption rate of concrete surface can be expected. But the key issue is how to make the two functions more adaptable when used on the surface.

Very recently, the development of the hybrid nanocomposites shows the potential of endowing concrete surface with low water absorption features. A series of core-shell structured hybrid nanocomposites, such as nanosilica/polymethylhydrosiloxane (NS/PMHS), ${ }^{17}$ polymethylhydrosiloxane-/nanosilica (PMHS/NS), ${ }^{18}$ tridecafluorooctyltriethoxysilane-/nanosilica (FAS/NS), ${ }^{19}$ were designed for surface treatment of hardened cement-based materials. The advantages of both normal organic and inorganic silica-based treatment agents are explored.

Morphology study shows that the core-shell structured nanocomposites can be formed by adjusting of the synthesizing parameters of the composites (Fig. 6). By applying the nanocomposites onto the surface of the hardened cement mortar, it shows that the pores of about 10-100 nm can be reduced (Fig. 7a), showing the compaction of the microstructure. Together with organic agent, a great reduction of
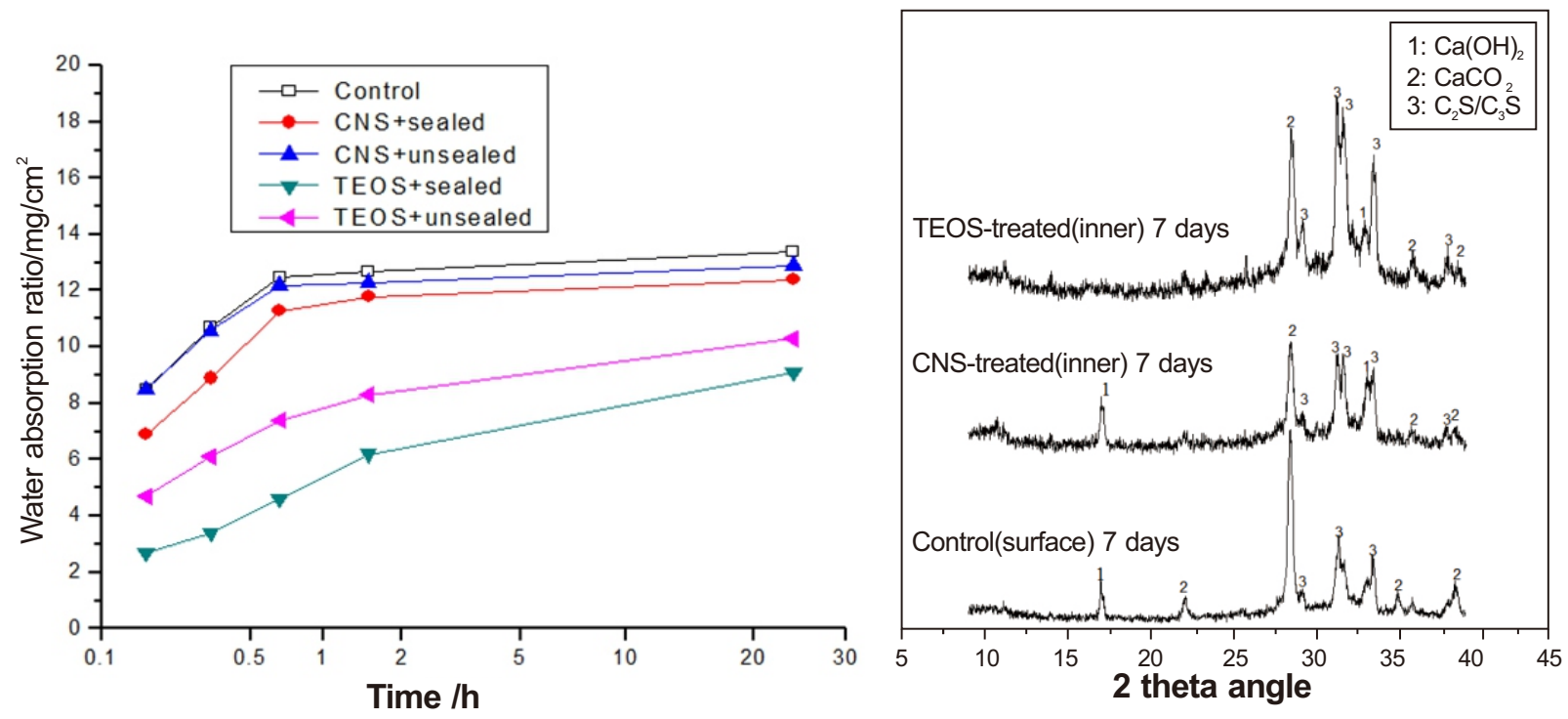

Fig. 5 Water absorption ratios of one-month old mortars cured for 14 days at different regimes (CNS, colloidal nanosilica) and XRD patterns of the inner hydrates of CNS/TEOS-treated samples. 
water absorption ratio (Figs. 6b, c) have been achieved. Moreover, the design and synthesizing of the organic/inorganic nanocomposite show the potential of adjusting the hydrophobic degree, as well as the anti- weathering capability of hybrid structured nanocomposites, which also provides a novel functionalization of cement concrete through the simple surface treatment technique.
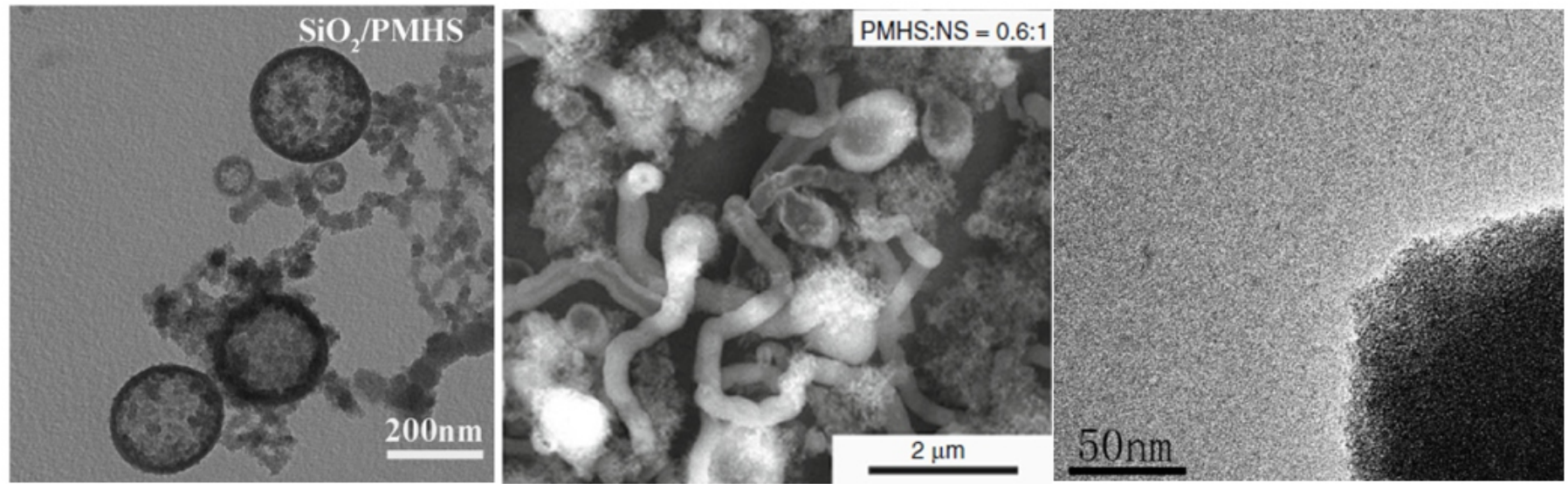

Fig. 6 Morphology of the NS/PMHS, PMHS/NS, and FAS/NS hybrid nanocomposites. ${ }^{17-19}$

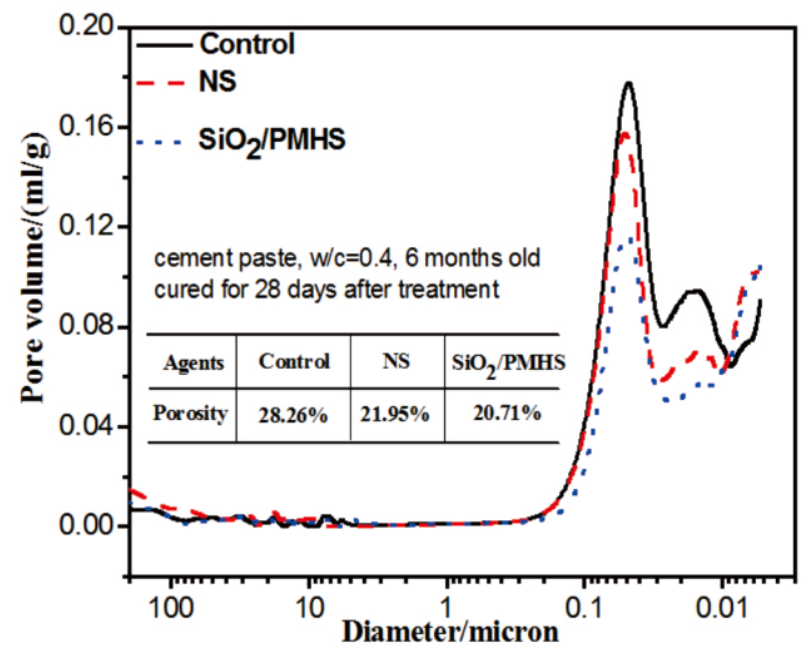

a)

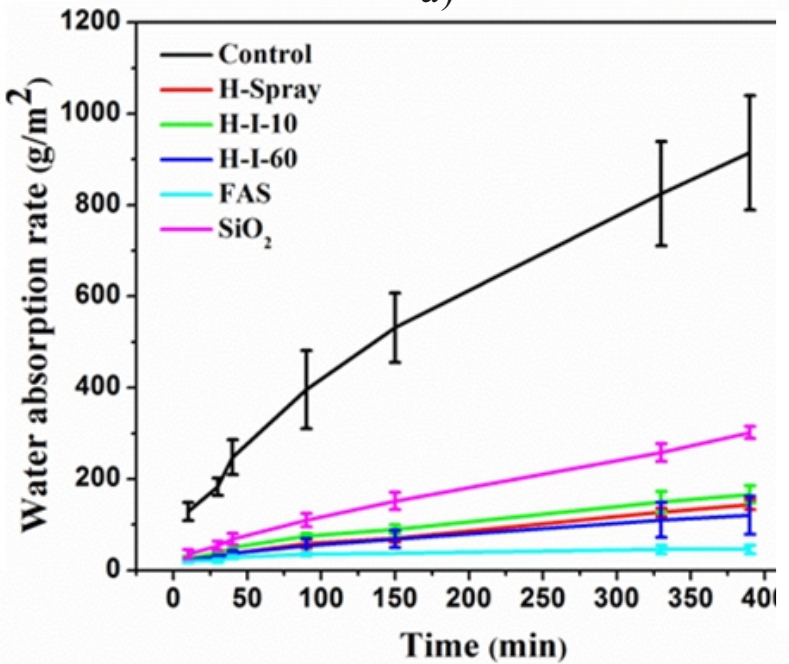

c)

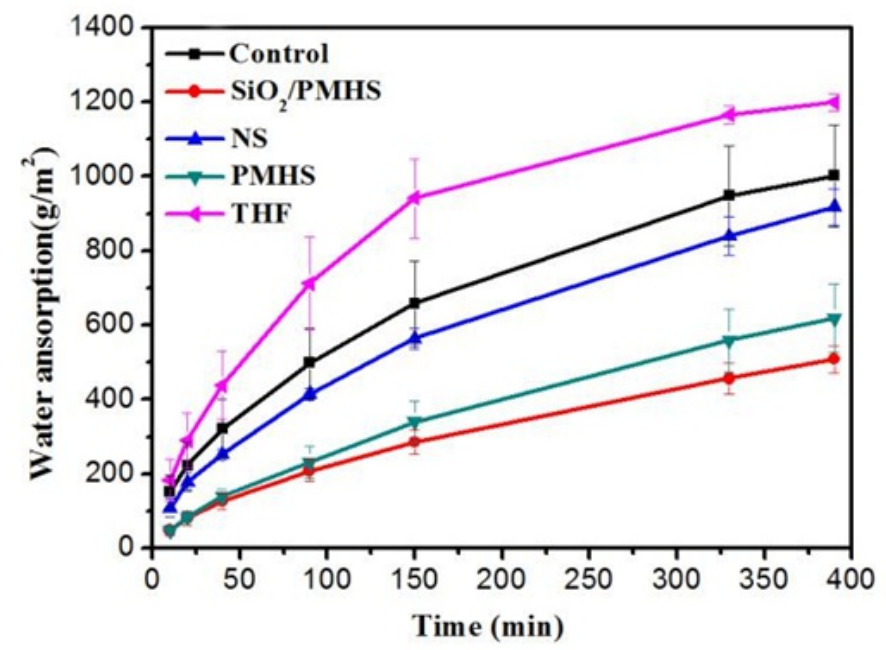

b)

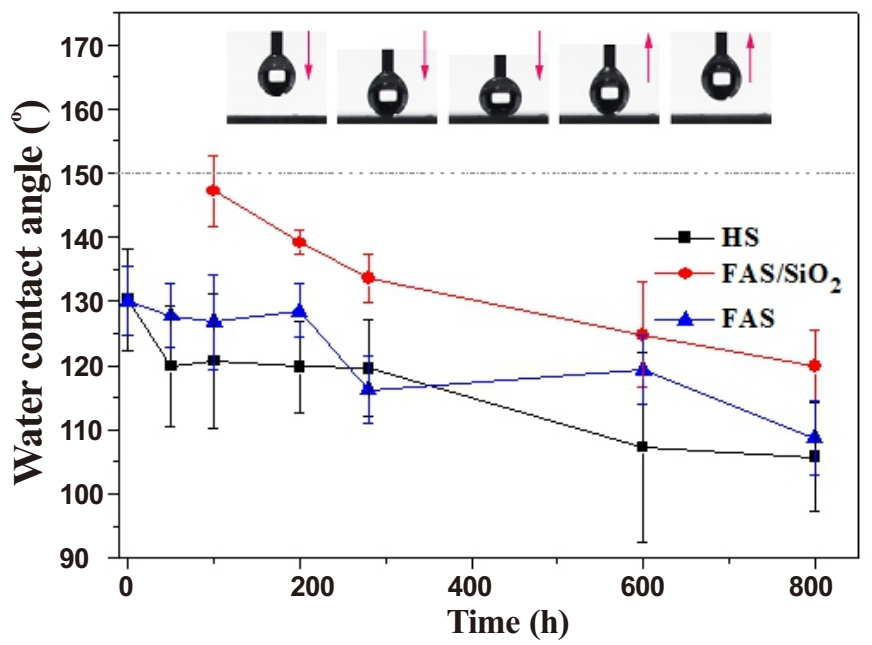

d)

Fig. 7 Performance of the hybrid nanocomposites for surface treatment (a: pore filling effect; b, c: reduction of water absorption of the hybrid nanocomposites-treated sample; $\mathrm{d}$ : anti-weathering performance of the organic/inorganic nanocomposites. 


\subsection{Surface coating with silica-based hybrid nanocomposites for visible light photocatalyst}

Surface treatment of concrete for multi-functions is a practical way of equipping concrete with additional functions. However, physical attachment of the treatment agent onto concrete surface would definitely result in a poor durability of the function. Thus, creating the bonding between the treatment agent with the hardened concrete is the key issue. The use of nanosilica for surface treatment shows the potential for improving the quality of surface concrete by taking advantages of the particle size and high reactivity features. Another feature of nanosilica is the high amount of hydroxyl group, which can be easily connected to other chemical groups, thus forming composites of multi-functions. It can be inferred that multi-functional cement concrete can be produced by the design and use of silica-based hybrid materials through surface treatment.

Photocatalysis is the most commonly researched feature of concrete with the desired applications of air purifying, self-cleaning, etc. Semiconductors, such as nanoTiO 2 have often been applied to enable materials with these properties. $\mathrm{NanoTiO}_{2}$ has been added into concrete to make a self-cleaning concrete structure. However, the huge amount of nanoTiO $\mathrm{Ti}_{2}$ required makes it impractical in large scale uses considering the price, therefore the surface treatment of concrete using nanoparticle is a more practical way. When applying photocatalysts for surface-treatment of concrete, two key issues are (1) high photocatalysis efficiency under visible light, and (2) good durability, which could be fading due to the loose bonding with the concrete matrix. To tackle these problems, specially designed $\mathrm{BiOBr} @ \mathrm{SiO}_{2}$ nanoparticles as shown in Fig. 8 were used. It can be seen that the core-shell structured nanostructures can be designed. Nanosilica could act as binder of the functional nanoparticles with concrete matrix, and making the surface more photocatalytical. $\mathrm{SiO}_{2}$ can reduce the release of photocatalysts on the surface of cement pastes after the simulated raining. The Fig. 8 shows that the cement pastes treated with $\mathrm{BiOBr} @ \mathrm{TiO}_{2}$ composites almost kept the superior photocatalytic property after curing for 3 months and simulated weathering process.

The innovative design of the silica-based core-shell nanocomposites for surface treatment makes concrete multi-functional, such as superhydrophobic, ${ }^{12,17,18}$ photocatalytic, ${ }^{20}$ microwave-absorption ${ }^{21}$ happen, leading to the new stage of development of nanoengineered concrete.

\section{Meta-concrete for energy harvesting and sensing}

\subsection{Thermoelectricity for waste heat harvesting}

Heat is the most earth-abundant energy source that can be directly converted into electrical energy using the thermoelectric (TE) effect. ${ }^{22}$
A good TE material should have high Seebeck coefficient and electrical conductivity to achieve higher power output, while the low thermal conductivity is desired to maintain the temperature difference across the material.

To explore the feasibility of developing concrete with TE behaviors, limited research has been performed using steel or carbon fiber in concrete to attempt to increase TE effect of concrete. ${ }^{23,24}$ For instance, Sun et al. studied the TE effect of carbon fiber-reinforced concrete (CFRC), and they discovered that the Seebeck coefficient has increased with the content of $\mathrm{CF}$ incorporation from $0.2-1.1 \mathrm{wt} \%{ }^{25} \mathrm{D}$. D. L. Chung et al. investigated the TE properties of CFRC with silica fume and latex admixtures. The results indicated that admixtures have a minor influence on the Seebeck effect. ${ }^{26}$ Wen et. al. used stainless steel fibers in cement paste and they discovered high electrical conductivity and low Seebeck coefficient with stainless steel fibers at $8 \mathrm{~m}^{26}$ However, none of these studies have investigated all three related TE properties and the Seebeck coefficient was too low to be used in practice.

The early research of using nanoparticles to improve TE effect of concrete was done by Lu's group in the use of $\mathrm{ZnO}$ and $\mathrm{Al}$ doped $\mathrm{ZnO}$ (AZO) nanoparticles with $20 \mathrm{~nm}$ in diameter. ${ }^{27}$ The results showed that after adding nanoparticles to the mixture, the Seebeck coefficient was increased to $17 \%$, thermal conductivity was decreased to $9 \%$, and electrical conductivity was increased to $37 \%$ compared to the corresponding values for plain cement paste, as shown in Fig. 8. The addition of $\mathrm{ZnO}$ nanoparticles improved the TE properties of cement paste which can be attributed to the formation of $\mathrm{Zn}(\mathrm{OH})_{2}$ and increased volume of pore solutions in $\mathrm{ZnO}$-cement paste. A similar work has resulted in a high Seebeck coefficient at $3400 \mathrm{~V} / \mathrm{C}$ at $5 \mathrm{wt} . \%$ of $\mathrm{ZnO}$ incorporation in cement matrix, ${ }^{28}$ which is an order of magnitude higher than that of CFRC.

One of recent study on carbon fiber reinforced concrete performed by Wei et al. showed that carbon fibers could enhance the thermoelectric effect of concrete. ${ }^{29}$ At $1.0 \mathrm{wt} \%$ of carbon fiber incorporation, the maximum power output was estimated at 4-5 $\mathrm{W}$ per square meter at the temperature difference about $62{ }^{\circ} \mathrm{C}$ with a $20 \mathrm{~mm}$ slab thickness.

In summary, current work has indicated that TE properties of cement can be enhanced by adding carbon fiber or metal oxides nanoparticles in cement matrix. Among them, metal oxides nanoparticles have resulted in higher voltage output due to the higher Seebeck coefficient. This suggests the resulting materials can potentially be used to convert stored thermal energy in concrete for power generation. However, more systematic investigations are needed to fully
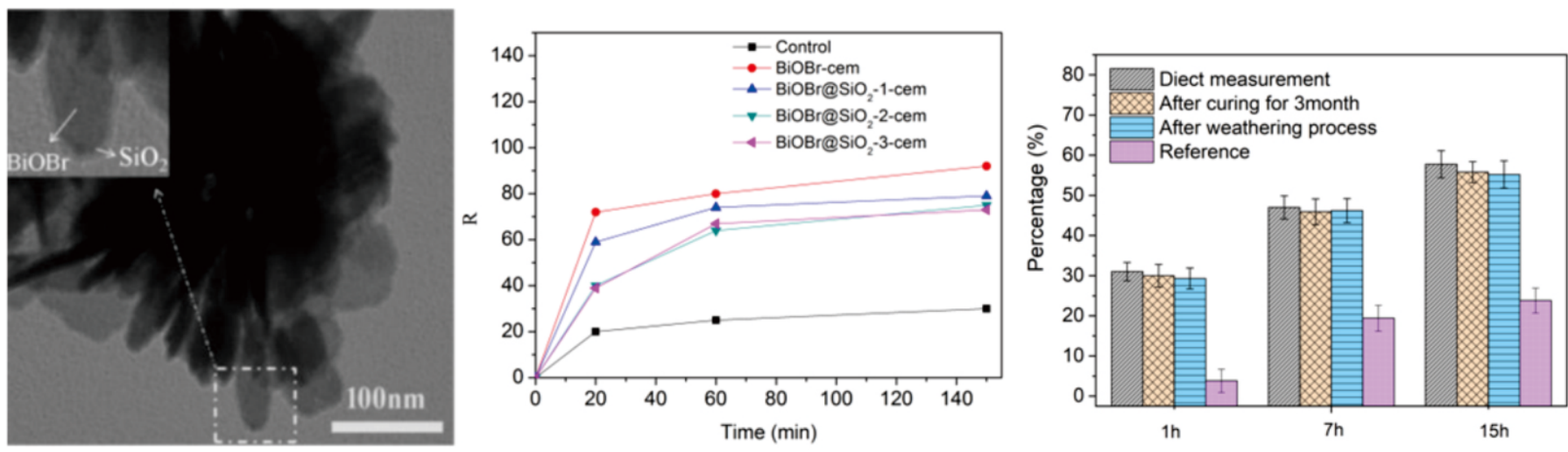

Fig. 8 TEM image of $\mathrm{BiOBr} @ \mathrm{SiO}_{2}(\mathrm{~L})$, removal percentage of $\mathrm{BiOBr}$ and $\mathrm{BiOBr} @ \mathrm{SiO}_{2}$ nanomaterials(M), and photocatalytic rhodamine $\mathrm{B}$ removal percentage after weathering $(\mathrm{R}) .^{20}$ 
explore the promises of TE concrete. More importantly, it is crucial to develop a standard testing method in order to eliminate the large variance of data generated by different studies.

\subsection{Piezoelectricity for mechanical energy harvesting in meta-} concrete

Piezoelectric materials can directly convert mechanical energy into electricity or vice versa. These materials are widely used as actuators, sensors, transducers and energy harvesters. Concrete structures are widely exposed to mechanical loading, deflection and vibration, which provides abundant opportunity for piezoelectric based concrete materials for energy harvesting and sensing applications. The most commonly used piezoelectric materials are piezoceramics, such as PZT (lead zirconatetitanate), due to their high electro-mechanical coupling properties, also known as the piezoelectric coefficients $\mathrm{d}_{\mathrm{i} \text {. }}$. However, the intrinsic brittleness of PZT restricts their applications in civil infrastructures. Compared to piezoceramics, piezo-based polymers have good piezoelectric properties under high strain conditions to harvest mechanical energy. Among the piezoelectric polymers, PVDF (polyvinylidene difluoride) is the most commonly used flexible piezoelectric materials due to its high flexibility, high piezoelectric properties, and good mechanical properties. The voltage coefficient of PVDF is 10 times higher than that of ceramic materials which makes it an exceptional candidate for energy harvesting and sensing applications. ${ }^{30,31}$

Due to the low dissipation factor and high permittivity, Jaitanonget al. studied the effect of using PVDF to enhance the poling of 0-3 PZT cementitious composites. ${ }^{32}$ It has been found that the composite with $5 \mathrm{vol} \%$ PVDF increased the $\mathrm{d}_{33}$ to $24 \mathrm{pC} / \mathrm{N}$ and $\mathrm{g}_{33}$ to $25.7 \times 10^{-3} \mathrm{Vm} / \mathrm{N}$, respectively Fig. 10a). Moreover, a significant reduction in poling time was discovered with PVDF addition due to the good bonding between PVDF and cement particles, as shown in Fig. $10 \mathrm{~b})$.
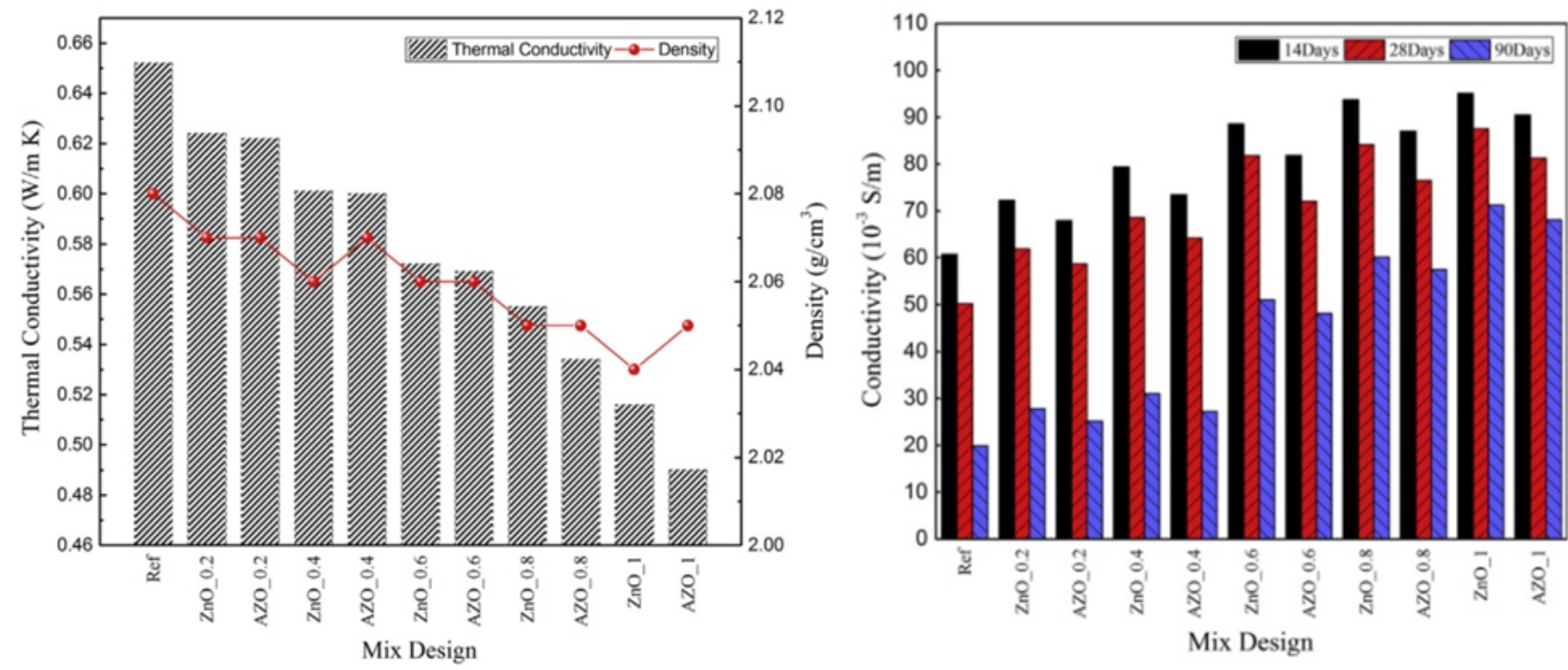

Fig. 9 Thermal conductivity of AZO-cement (left) and electrical conductivity with various mix design on AZO-cement (right).
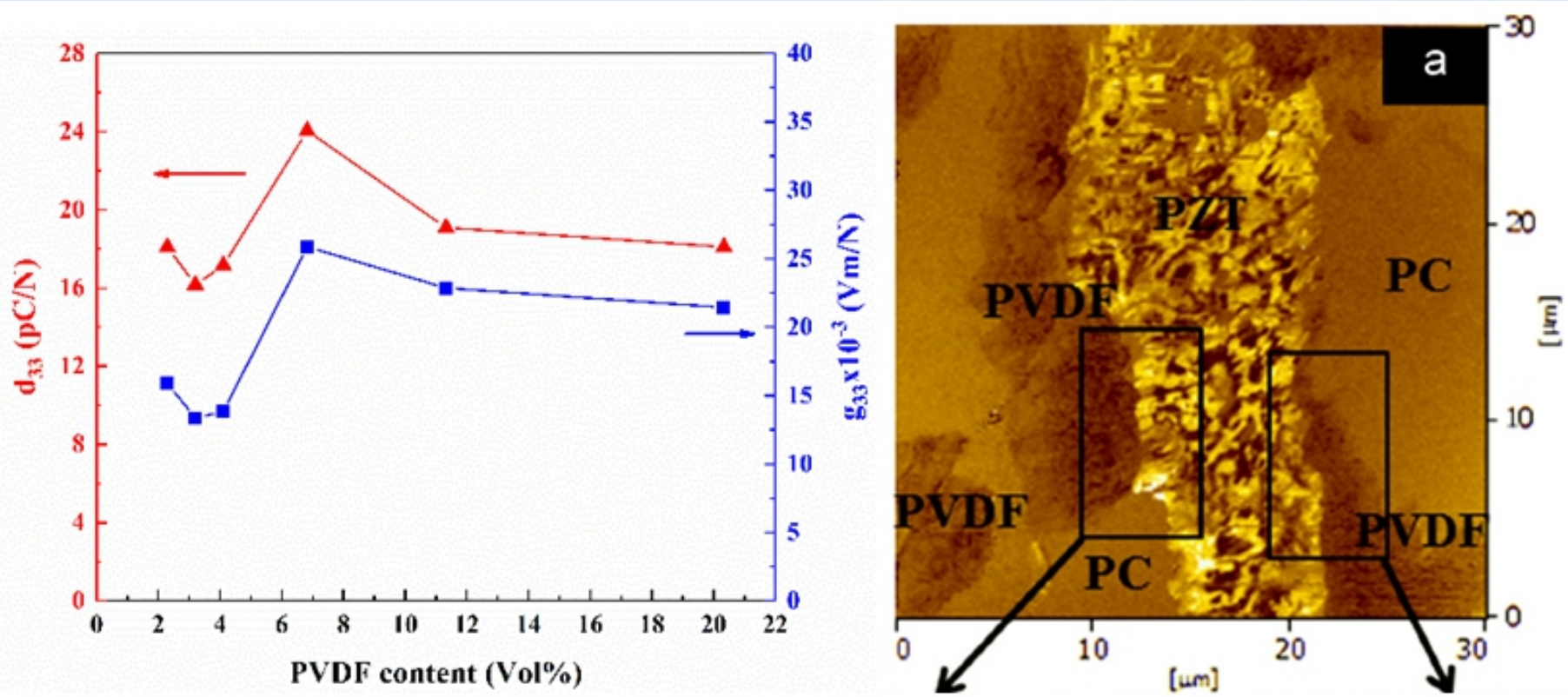

Fig. 10 Piezoelectric charge coefficient $\left(\mathrm{d}_{33}\right)$ and piezoelectric voltage coefficient $\left(\mathrm{g}_{33}\right)$ of PC/PVDF/PZT cement composite (left); piezo-response image of samples (right). ${ }^{32}$ 
In a recent study, Lu's group has discovered the feasibility of using PVDF based nano-generator to harvest mechanical energy from engineering cementitious composites (ECC). ${ }^{33}$ The experiment was conducted by four-point bending tests coupled with real-time monitoring of the voltages generated by PVDF nano-generator, Fig. 11a). The favorable voltage output has reached up to around $17 \mathrm{mV}$, as shown in Fig. 11b).

\subsection{Piezoelectric concrete for sensing}

To address the incapability of piezoceramics (PZT) with concrete, researchers have fabricated several types of cement-based piezoelectric composites which can be encapsulated into concrete. ${ }^{34,35}$ They have used PZT nano-powders through sol-gel process to synthesize the 0-3 piezoelectric cementitious composites. ${ }^{34}$ The numbers " $\mathrm{m}-\mathrm{n}$ " indicate the connectivity between piezoceramic (m) and cement matrix (n) phases. For instance, 0-3 means the piezoceramic particles mixed in cement paste as Fig. 12 shown. In this study, a high piezoelectric coefficient $\mathrm{d}_{33}$ was reached at $53.7 \mathrm{pC} / \mathrm{N}$, for the composites with 80 vol\% PZT nano-powder incorporations. The favorable piezoelectric effect was attributed to high crystallinity of PZT nanoparticles and their net-work distributions in the cement composites.

A further study was conducted by the same group using 1-3 cement-based piezoelectric composites as a broadband transducer for structural health monitoring. The PZT-cement transducers were embedded in concrete beams to perform active and passive sensing of damage zone using acoustic emission method. Crack localization and damage evolutions were successfully detected using both methods as shown in Fig. 12. This work has shown a great promise of using PZTcement based transducer for structural health monitoring. ${ }^{35}$

Cheng et al fabricated various cement based piezoelectric composites by using sulfoaluminate cement as matrix. ${ }^{36}$ The results indicate that the piezoelectric strain factor $d_{33}$ increases as the piezoelectric volume fraction increases. In the frequency range of 40$100 \mathrm{kHz}$, the dielectric constants of the composites decrease sharply, which is mainly attributed to interfacial polarization in the composite. Lam et al fabricated piezoelectric cement-based 1-3 composites with different volume fractions of PZT ranging from 0.25 to 0.77 . It was
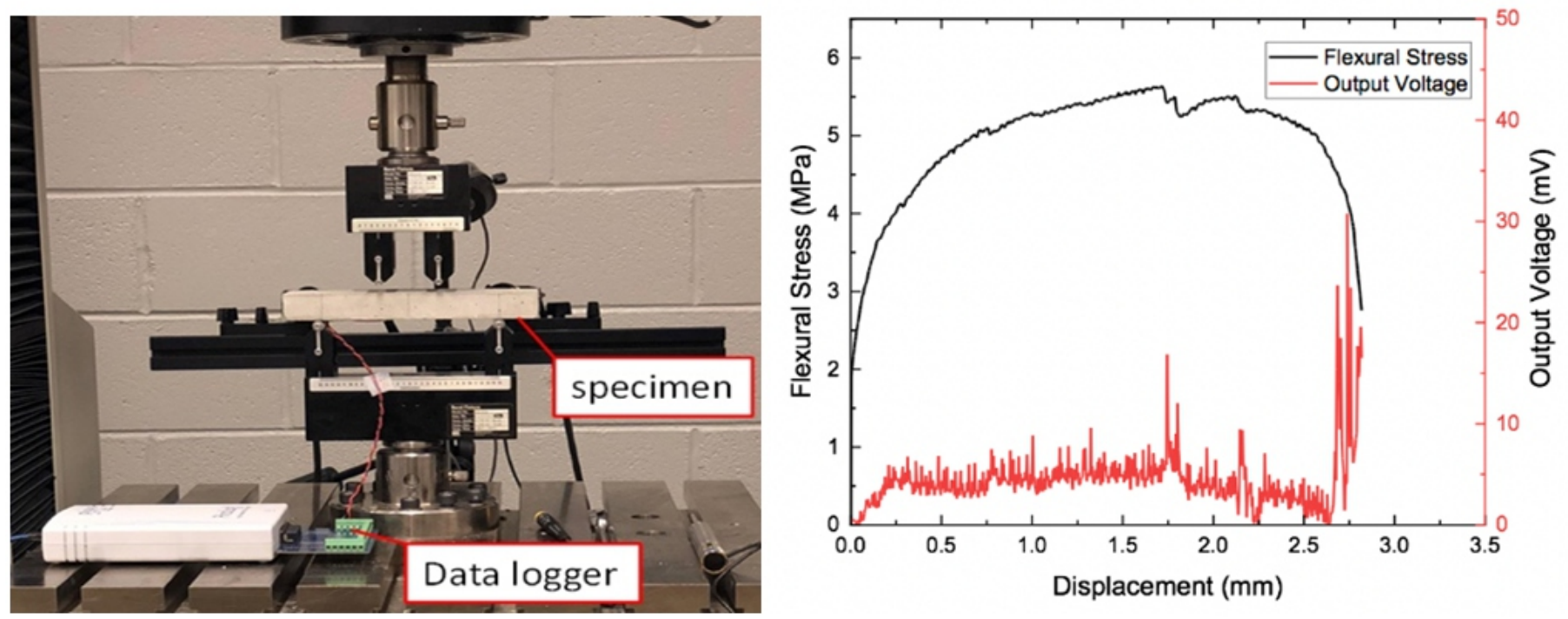

Fig. 11 The experimental setup for 4-pt bending test of piezoelectric cementitious composite (left) and the flexural stress-output voltage-displacement curve of the sample with $0.5 \mathrm{~mm} / \mathrm{s}$ loading rate (right).

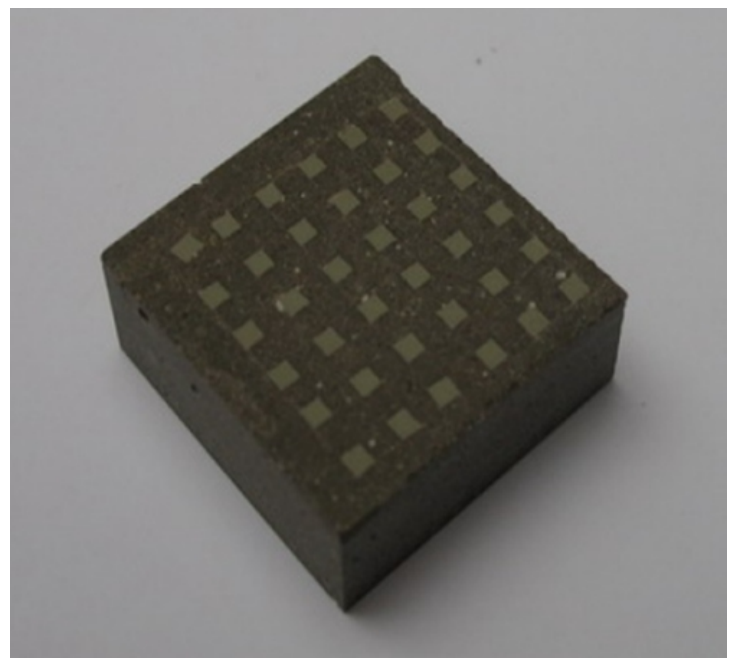

Fig. 12 Sample of 1-3 cement-based piezoelectric composites. ${ }^{35}$ 
found that the 1-3 composites have good piezoelectric properties, and the thickness electromechanical coupling coefficient could reach 0.55 in the composite with a ceramic volume fraction of 0.25 . $^{37}$

Built on previous studies of PZT-cement composites, Banerjee et $a l .^{38}$ has examined the effect of using conductive metal nanoparticles (Al with $18 \mathrm{~nm}$ ) on dielectric and piezoelectric properties of PZTcement composites. Significant increase in both dielectric and piezoelectric properties were observed on PZT-cement with Al nanoparticles. However, the performance was degraded after 2 days which can be attributed to the switching of PZT dipoles and remnant polarization stabilized over time. To improve the polarization of PZTcement composites, Zhao et al. dispersed a small amount of carbon nanotubes (CNTs) in the cement-sand- based piezoelectric composites ranging from 0 to $0.9 \mathrm{vol} \%{ }^{39}$ The maximum $\mathrm{d}_{33}$ value of $13.0 \mathrm{pC} / \mathrm{N}$ was reached at $0.6 \mathrm{vol} \% \mathrm{CNT}$ incorporation, the percolation threshold of CNTs. The dielectric constant was increased significantly with the increase of CNTs content due to increased electrical conductivity. The sensing performance of CNT-PZT-cement was also investigated under compressive loading at $0-4.0 \mathrm{~Hz}$. A linear behavior of stress-voltage relations was observed as the highlight of the work, which indicated the promises of using this piezoelectric cement composites for sensing and structural monitoring.

\section{Summary}

In this paper, we have discussed the recent advances of using nanotechnology to develop meta-concrete with unique properties, including high modulus of elasticity, improved water resistivity, high photocatalytic, and thermoelectric and piezoelectric for energy harvesting and sensing. Promising results from these studies suggested a great potential of using meta-concrete with novel functionality for a broad range of applications.

Despite the recent advancement, developing meta-concrete with unique multifunctionalities are still in their infancy. Many scientific and technological challenges need to be addressed in the future studies. For instance, how to keep the balance of materials cost, function, performance and workability? How to improve the efficiency? Regardless, the scientific and technological importance of developing meta-concrete is evident, and their potential impact is remarkable to the design, construction and testing of future infrastructures. Fortunately, the rapid revolution in nanotechnology and processing techniques are opening up exciting venues to create meta-concrete through additive manufacturing, modular construction, and artificial intelligent design and construction. We hope this article may pave the road for the future development of meta-concrete.

\section{Acknowledgement}

Dr. Na Lu would like to thank the generous support from US National Science Foundation CAREER Program (under the grant of CMMI1560834).

\section{References}

1. M. S. M. Norhasri, M. S. Hamidah and A. M. Fadzil, Constr. Build. Mater, 2017, 133, 91-97.

2. 2016, US9499439B2.

3. 2015, US9365456B2.
4. M. S. Konsta-Gdoutos, Z. S. Metaxa and S. P. Shah, Cem. Concr. Compos., 2010, 32, 110-115.

5. S. P. Shah and M. S. Konsta-Gdoutos, Concr. Int., 2017, 39, 37-42.

6. Z. S. Metaxa, M. S. Konsta-Gdoutos and S. P. Shah, in American Concrete Institute, ACI Special Publication, 2009, 267, 11-20.

7. P. Mondai, S. Shah and L. Marks, ACI Mater, 2008, 105, 174-179.

8. J. M. Makar and G. W. Chan, J. Am. Ceram. Soc., 2009, 92, 1303-1310.

9. P. A. M. Basheer, L. Basheer, D. J. Cleland and A. E. Long, Constr. Build. Mater., 1997, 11, 413-429.

10. C. Christodoulou, C. I. Goodier, S. A. Austin, J. Webb and G. K. Glass, Constr. Build. Mater., 2013, 48, 708-716.

11. H. E. Cardenas and L. J. Struble, J. Mater. Civ. Eng., 2006, 18, 554-560.

12. P. Hou, X. Cheng, J. Qian and S. P. Shah, Constr. Build. Mater, 2014, 53, 66-73.

13. X. Pan, Z. Shi, C. Shi, T. C. Ling and N. Li, Constr. Build. Mater, 2017, 133, $81-90$.

14. P. Hou, R. Zhang, Y. Cai, X. Cheng and S. P. Shah, Constr. Build. Mater, 2016, 113, 890-896.

15. Z. Guo, P. Hou, S. Huang, N. Xie, X. Cheng, L. . Singh, S. Valeriya and N. Viktoriya, Ceramics-Silikáty, 2018, 62, 332-341.

16. D. Wang, University of Jinan, 2018.

17. R. Li, P. Hou, N. Xie, Z. Ye, X. Cheng and S. P. Shah, Cem. Concr. Compos., 2018, 87, 89-97.

18. P. Hou, R. Li, H. Li, N. Xie, X. Cheng and L. P. Singh, J. Therm. Anal. Calorim., 2018, 134, 1775-1784.

19. P. Hou, R. Li, Q. Li, N. Lu, K. Wang, M. Liu, X. Cheng and S. Shah, ES Mater. Manuf., 2018, 1, 57-66.

20. D. Wang, P. Hou, P. Yang and X. Cheng, Appl. Surf. Sci., 2018, 430, 539-548.

21. D. Wang, P. Yang, P. Hou, L. Zhang, X. Zhang, Z. Zhou, N. Xie, S. Huang and X. Cheng, Cem. Concr. Compos., 2017, 80, 268-276.

22. Y. Feng, A. Elquist, Y. Zhang, K. Gao, I. Ferguson, A. Tzempelikos and N. Lu, Compos. Part B Eng., 2019, 156, 108-112.

23. S. Wen and D. D. . Chung, Cem. Concr. Res., 2002, 32, 821-823.

24. S. Wen and D. D. L. Chung, J. Mater. Sci., 2004, 39, 4103-4106.

25. M. Sun, Z. Li, Q. Mao and D. Shen, Cem. Concr. Res., 1998, 28, 1707-1712.

26. S. Wen and D. D. . Chung, Cem. Concr. Res., 1999, 29, 1989-1993.

27. S. A. Ghahari, E. Ghafari and N. Lu, Constr. Build. Mater, 2017, 146 755-763.

28. T. Ji, X. Zhang and W. Li, Constr. Build. Mater, 2016, 115, 576-581.

29. J. Wei, Z. Nie, G. He, L. Hao, L. Zhao and Q. Zhang, RSC Adv., 2014, 4, 48128-48134.

30. D. Y. Xu, L. Qin, S. F. Huang and X. Cheng, Adv. Mater. Res., 2011, 306-307, 839-843.

31. J. Xu, M. J. Dapino, D. Gallego-Perez and D. Hansford, Sensors Actuators A Phys., 2009, 153, 24-32.

32. N. Jaitanong, R. Yimnirun, H. R. Zeng, G. R. Li, Q. R. Yin and A. Chaipanich, Mater. Lett., 2014, 130, 146-149.

33. Y. F. Su, R. R. Kotian and N. Lu, Compos. Part B Eng., 2018, 153, 124-129.

34. Z. Li, H. Gong and Y. Zhang, Curr. Appl. Phys., 2009, 9, 588-591.

35. L. Qin, S. Huang, X. Cheng, Y. Lu and Z. Li, Smart Mater. Struct., 2009, 18, 095018.

36. C. Xin, H. Shifeng, C. Jun and L. Zongjin, J. Appl. Phys., 2007, 101, 094110

37. K. H. Lam and H. L. W. Chan, Appl. Phys. A, 2005, 81, 1451-1454.

38. S. Banerjee, J. Torres and K. A. Cook-Chennault, Ceram. Int., 2015, 41, 819-833.

39. P. Zhao, S. Wang, A. Kadlec, Z. Li and X. Wang, Ceram. Int., 2016, 42, $15030-15034$.

Publisher's Note Engineered Science Publisher remains neutral with regard to jurisdictional claims in published maps and institutional affiliations. 